\title{
Pseudoaneurysm overlying an osteochondroma: a noteworthy complication
}

\author{
Celeste Scotti • Enrico M. Marone - Laura E. Brasca • Giuseppe M. Peretti • \\ Roberto Chiesa - Alessandro Del Maschio - Gianfranco Fraschini · \\ Francesco Camnasio
}

Received: 29 May 2009/Accepted: 19 October 2010/Published online: 20 November 2010

(C) The Author(s) 2010. This article is published with open access at Springerlink.com

\begin{abstract}
Pseuodaneurysms are an extremely rare complication of osteochondromas. We describe a case of traumatic pseudoaneurysm of the brachial artery presenting as a soft tissue mass in a patient who was treated for an osteochondroma 3 years earlier. This case demonstrates that radiographic follow-up of large osteochondromas is mandatory and that, in patients with soft tissue masses and a history of osteochondroma, pseudoaneurysms should be included in the differential diagnosis.
\end{abstract}

Keywords Pseudoaneurysm - Osteochondroma .

Soft tissue mass · Brachial artery

\section{Introduction}

Osteochondromas are hamartomas derived from an aberrant subperiosteal germ of the physeal cartilage and grow by normal enchondral ossification [1]. They are the most

C. Scotti · G. M. Peretti - G. Fraschini · F. Camnasio ( $₫)$

Department of Orthopaedics and Traumatology,

San Raffaele Scientific Institute, Via Olgettina 60,

20132 Milan, Italy

e-mail: camnasio.francesco@hsr.it

E. M. Marone - R. Chiesa

Department of Vascular Surgery,

San Raffaele Scientific Institute,

Via Olgettina 60, 20132 Milan, Italy

L. E. Brasca - A. Del Maschio

Department of Radiology, San Raffaele Scientific Institute,

Via Olgettina 60, 20132 Milan, Italy

G. M. Peretti

Faculty of Exercise Sciences, University of Milan,

Via Kramer 4/A, 20129 Milan, Italy common benign tumors of bone, accounting approximately for one third of benign lesions of the skeleton [2]. Nevertheless, as they are usually asymptomatic, the real incidence is probably higher [3]. Patients usually present during the second decade of life [1]. Malignant transformation has been reported as a very rare complication, accounting for $<1 \%$ and occurring after puberty more frequently in the limb girdles $[1,4,5]$. In cases of hereditary multiple osteochondromas, the risk of sarcomatous change is higher $[1,6,7]$.

The radiographic appearance of this tumor is often diagnostic: as a matter of fact, the continuity of the lesion with the underlying native bone cortex and medullary canal is pathognomonic of osteochondroma. Computed tomography (CT) and magnetic resonance (MR) imaging are useful for identifying and studying complications such as malignant transformation, bone fracture, vascular compromise, neurologic sequelae, and overlying bursa formation. The thickness of the cartilage cap is typically $1-3 \mathrm{~cm}$ in young patients, whereas in adults, it is often only a few millimeters thick or entirely absent [4, 8-10]. A continuously growing lesion and a hyaline cartilage cap $>1.5-\mathrm{cm}$ thick, after skeletal maturity, suggest malignant transformation. Cortical bone is devoid of mobile protons and shows low signal intensity in all pulse MR sequences, whereas the medullary component has the appearance of yellow marrow. MR imaging is the best radiologic modality for visualizing the effect of the lesion on surrounding structures and evaluating the hyaline cartilage cap. The high water content in nonmineralized portions of the cartilage cap has an intermediate to low signal intensity on T1-weighted MR images and very high signal intensity on T2-weighted images. These features typically allow accurate measurement of the cartilage cap thickness and distinction from overlying muscle on MR images [4, 11-13]. 


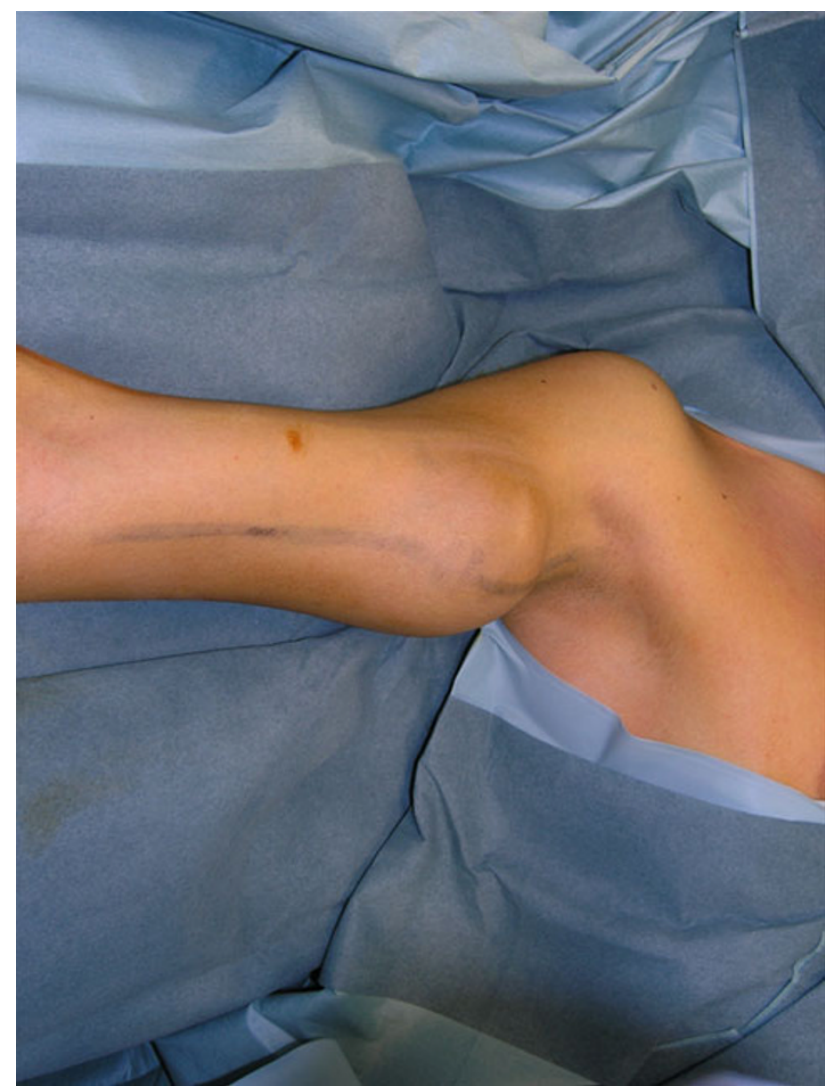

Fig. 1 Preoperative clinical image

Surgical excision is limited to symptomatic osteochondromas; as a matter of fact, when the tumor is asymptomatic, surgery is not recommended [1,14]. Indications for surgical resection are pain, deformity, compression to a neurovascular bundle, continuous growth, and suspected malignant transformation [1, 14, 15]. However, when a child presents with a large osteochondroma close to a neurovascular bundle, surgery is indicated to avoid further enlargement, which would make surgery more challenging with a higher risk of neurovascular complications. Pseudoaneurysm represents an extremely rare complication of osteochondroma and typically involves the popliteal and femoral arteries [16, 17]. We present a case of posttraumatic pseudoaneurysm of the brachial artery occurring after incomplete resection of an osteochondroma and presenting as a soft tissue mass.

\section{Case report}

A 14-year-old boy referred to our department for a slowly growing, nonpainful swelling in the medial upper right arm that he had noticed for 8 months. He had undergone partial resection of an osteochondroma in the posterior aspect of the proximal humerus 3 years earlier. Follow-up was unremarkable. He recalled a blunt trauma in his right upper arm while riding a horse; however, swelling presented some weeks later and was characterized by a gradual enlargement. An X-ray was performed and showed a large sessile osteochondroma in the median side of the proximal humerus. An MRI was performed to study the cartilaginous cap of the osteochondroma for malignant transformation and the soft tissues for a soft tissue tumor. MRI was considered suggestive of a soft tissue tumor, and the patient was referred to our department. Physical examination demonstrated a firm, nonpulsatile, $8-\mathrm{cm}$ swelling in the medial upper arm that was painless during palpation (Fig. 1). There were no signs of vascular or neurologic failure. No enlarged lymph nodes were palpable in the axilla. Bone scan revealed increased uptake at the site of the osteochondroma. MRI performed in our Institute revealed a large, complex mass close to the osteochondroma and the humeral artery. The nonhomogeneous lamellar structure of the mass on MRI, the hyperintensity on T1-weighted sequences, and the regularly capsulated aspect of the contours were suggestive of hematoma-thrombosis (Figs. 2a-c, 3a). Due to these characteristics, the hypothesis of malignant transformation was difficult to asses. The MR angiography facilitated the diagnosis, as it showed a pseudoaneurysm arising from the compressed brachial artery with extravasation of contrast
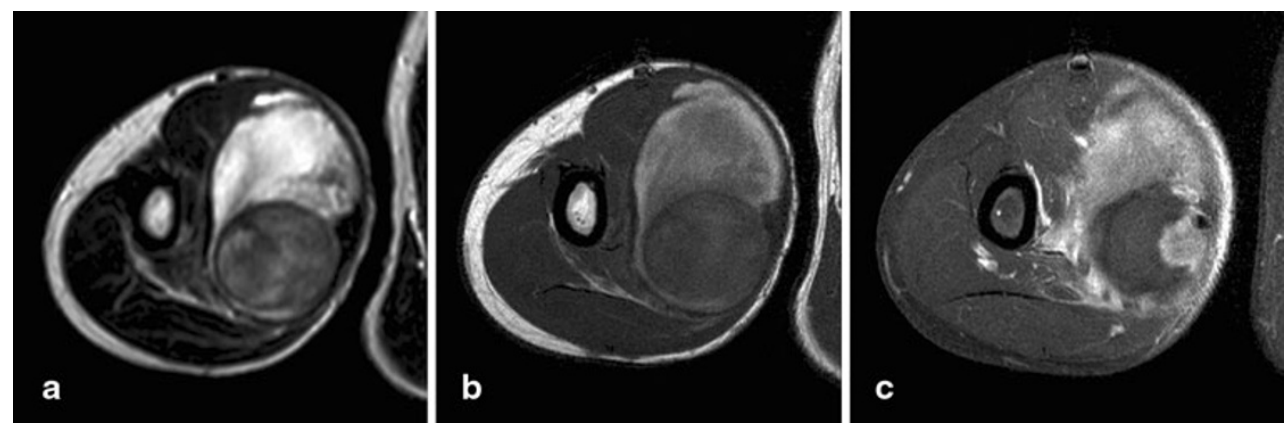

Fig. 2 Axial magnetic resonance image (MRI) T2 (a), T1 (b) and T1 turbo spin echo (TSE) fat-saturation (c) sequences. The mass has inhomogeneous signal on T2- (a) and T1- (b) weighted sequence, concentric aspect, regular contours (a, b) and did not diffusely enhance due to the presence of pseudoaneurysm, thrombus, and hematoma (c) 

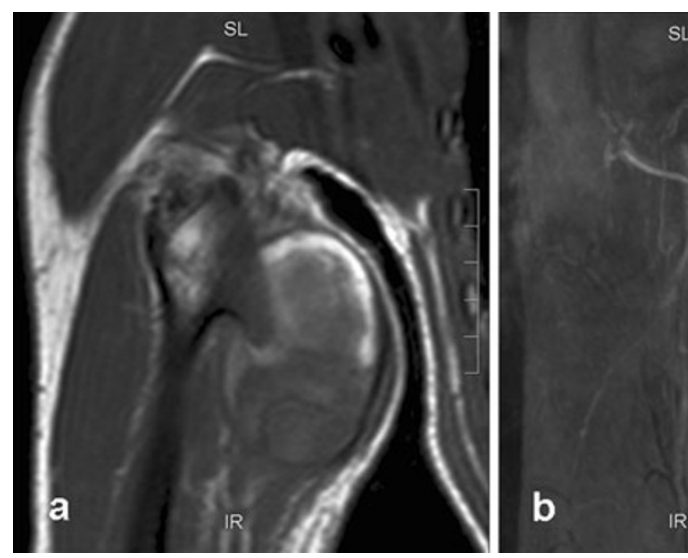

Fig. 3 Coronal magnetic resonance imaging (MRI) T1 turbo spin echo (TSE) (a), MR angiography (b), and digital angiography (c). MR angiography (b) identifies the pseudoaneurysm arising from the compressed brachial artery, with extravasation of contrast medium into the mass, well demonstrated also by the angiography (c)
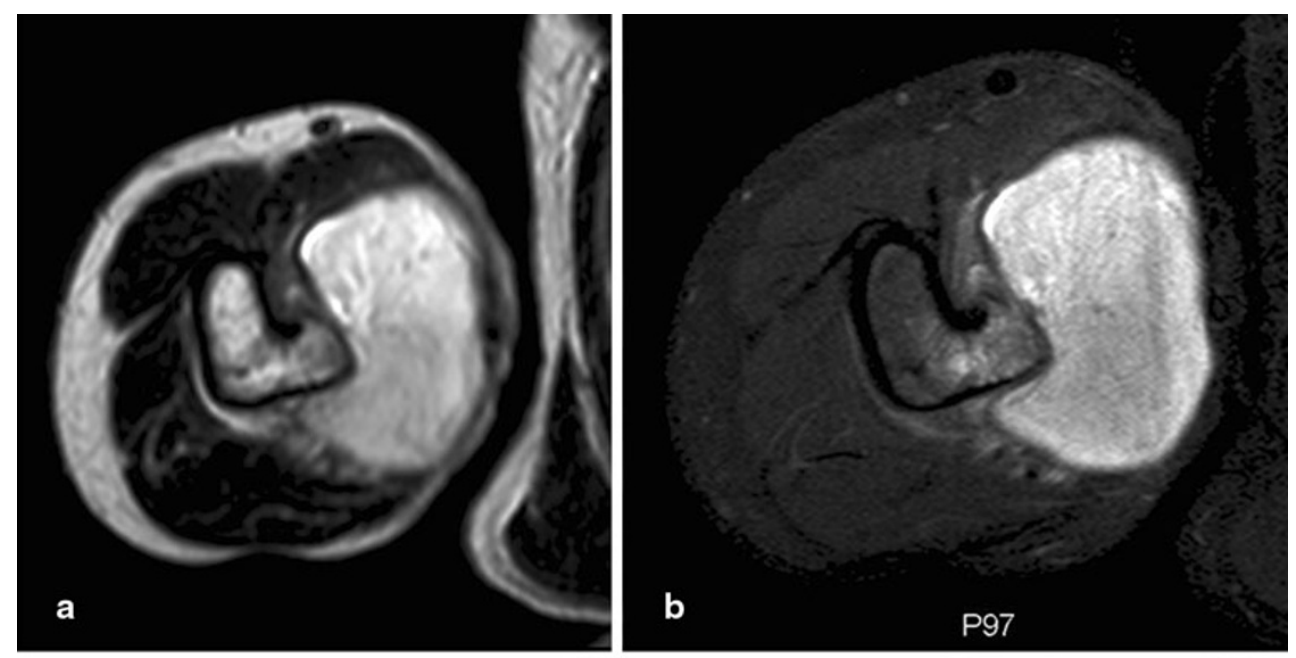

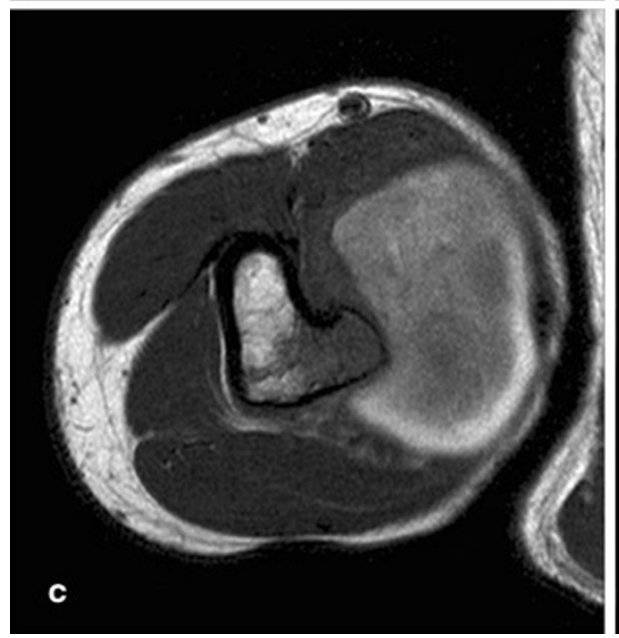

Fig. 4 Axial magnetic resonance imaging (MRI) T2 (a), protondensity fat saturation (PD fat-sat) (b), T1 turbo spin echo (TSE) (c), T1-TSE fat-sat (d) sequences of the osteochondroma underlying the

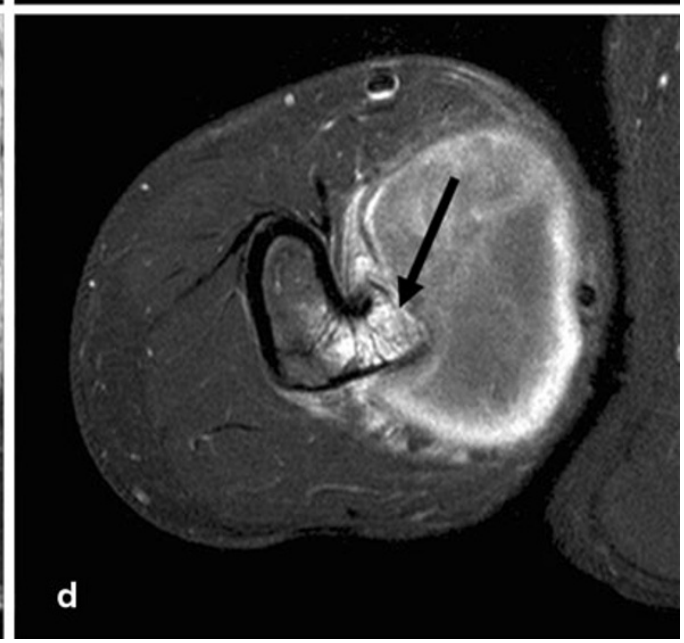

pseudoaneurysm. Note hyperintensity of the medulla adjacent to the lesion on PD sequences (b) and enhancement of the medulla itself after contrast media (arrow) (d) 

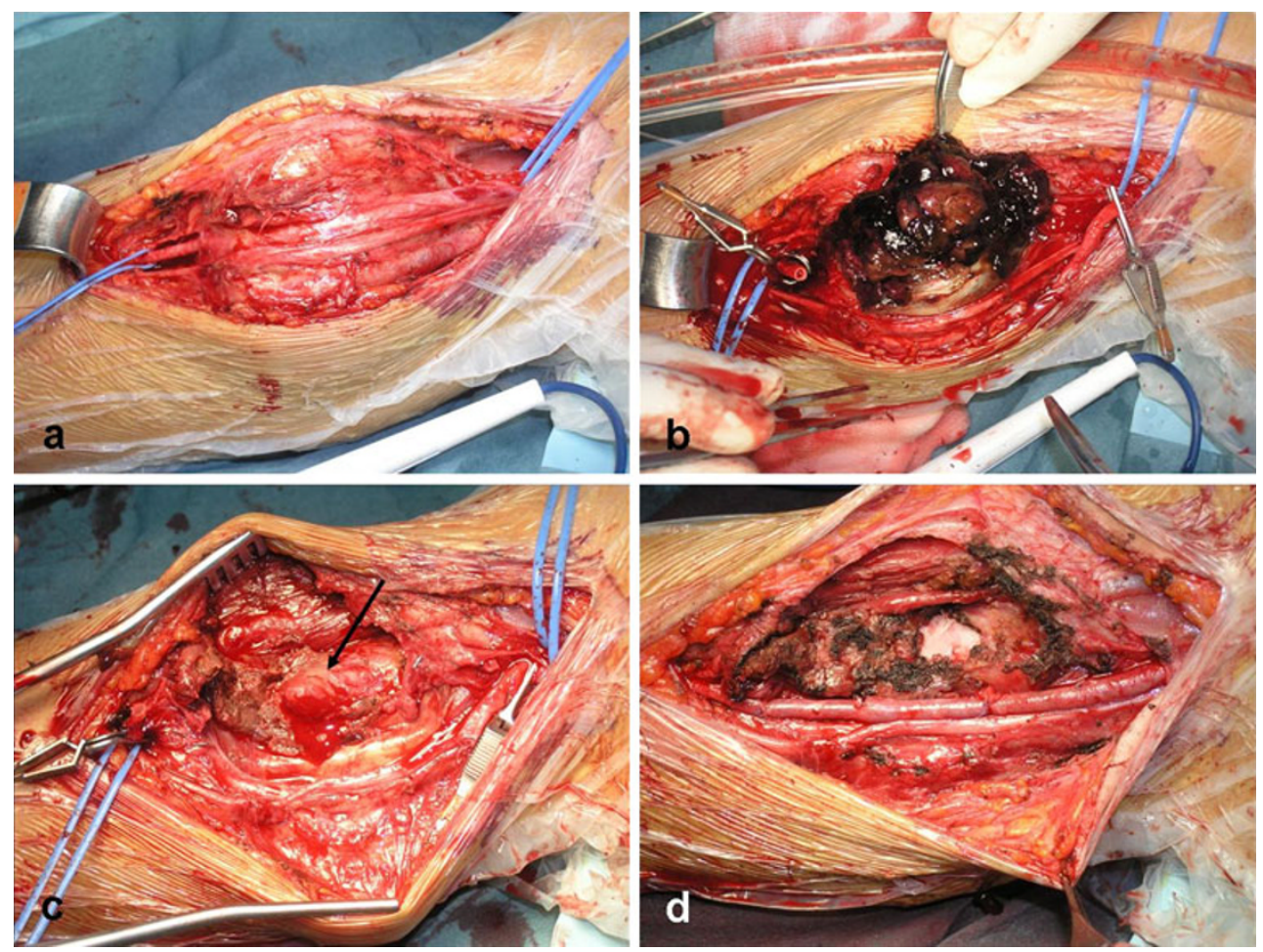

Fig. 5 Intraoperative images $(\mathbf{a}-\mathbf{c})$. The brachial artery and the pseudoaneurysm were isolated and the artery was controlled with nylon tapes (a); the pseudoaneurysmal sac was opened and the thrombus removed (b); a $7-\mathrm{cm}$ arterial segment including the

medium into the mass (Fig 3b). Angiography confirmed the diagnosis of pseudoaneurysm measuring $35 \mathrm{~mm}$ of patent lumen and provided a useful map for surgical planning (Fig. 3c). On T2-weighted MRI, an aspect of concentric rings was observed due to the presence of thrombus into the pseudoaneurysm that did not diffusely enhance with contrast material. In this case, the difficulty of recognizing the cartilage cap on $\mathrm{T} 2$ sequences because of the overlying pseudoaneurysm, hyperintensity on fat-suppressed proton density (PD)-weighted sequences, and enhancement of the medulla after contrast media could not exclude malignant transformation (Fig. 4a-d). Therefore, surgical exploration was mandatory. At operation, a longitudinal incision was made on the right medial upper arm. The nerve was identified and preserved. The brachial artery was controlled above and below the pseudoaneurysm with nylon tape (Fig. 5a). The pseudoaneurysm sac was opened and the thrombus removed (Fig. 5b). After retrograde flow was restored, a $7-\mathrm{cm}$ arterial segment that included the pseudoaneurysmal region was resected, and marginal resection of the osteochondroma was performed (Fig. 5c, d). Reversed saphenous vein interposition graft was then performed (Fig. 5d). The wound was closed and a suction drain was left in situ. At the end of the procedure, there were good radial and ulnar pulses palpable. Specimens of the sac wall pseudoaneurysm was resected, highlighting the underlying cartilaginous spur (arrow) of the osteochondroma, which probably caused the pseudoaneurysm (c); the osteochondroma was resected, and a reversed saphenous vein interposition graft was then performed (d)

and thrombus were negative following histopathological and microbiological examination. Histopathological diagnosis was osteochondroma. The patient was discharged and followed up in the outpatient clinic. Patient gave consent to the publication of this report.

\section{Discussion}

Pseudoaneurysms are a rare complication of osteochondromas and they are more frequent after the end of the enchondral ossification phase, when the cartilaginous cap become bony and sharp [17]. A hard swelling is the most common pattern of clinical presentation [17]. The most frequent site of this complication is the distal femur, whereas the arm is an extremely rare site, with only a few cases of pseudoaneurysm of the brachial artery reported in the literature [18-21]. Repair of the arterial injury is seldom possible. When an end-to-end anastomosis cannot be performed, graft replacement with the autologous saphenous vein or with a synthetic graft is preferred [17]. As a matter of fact, in our case, the extent of the pseudoaneurysm made the brachial artery non-reparable, and the vascular surgeon opted for a reconstruction with a saphenous vein graft. 
The case presented here is quite unusual, but the clinical presentation reflects the typical case found in the literature: an adolescent male, already treated for a large osteochondroma in an area with high risk of neurovascular compression, reporting a sport-related injury. The differential diagnosis included malignant transformation and Ewing's sarcoma, so we performed contrast-enhanced MRI and angiographic MRI to rule out these diagnoses. MRI is mandatory for evaluating such atypical masses in children and adolescents [22]. Moreover, malignant transformation is extremely rare in adolescence [1], and Ewing's sarcoma often presents with other signs and symptoms, such as fever, increased serum lactate dehydrogenase and erythrocyte sedimentation rate, leukocytis, and anemia [23], which were not present in our patient. Therefore, according to the patient's history of blunt trauma, to MRI features, and to the surgical findings of close proximity of the pseudoaneurysm to a cartilaginous spur, the diagnosis was traumatic pseudoaneurysm caused by an osteochondroma. This case shows how important it is to perform adequate resection of a large osteochondroma arising in close proximity to neurovascular structures. On the other hand, osteochondromas in adolescence may spontaneously regress [15] or recur, even after adequate resection, so that a radiographic follow-up is mandatory [1].

In conclusion, we emphasize the importance of correct treatment and follow-up of large osteochondromas in dangerous regions in order to avoid such complications. We also encourage the orthopedic oncologist to include pseudoaneurysms in the differential diagnosis of soft tissue masses, especially when there is a history of osteochondroma resection.

\section{Conflict of interest None.}

Open Access This article is distributed under the terms of the Creative Commons Attribution Noncommercial License which permits any noncommercial use, distribution, and reproduction in any medium, provided the original author(s) and source are credited.

\section{References}

1. Campanacci M (1999) Exostosis. In: Campanacci M (ed) Bone and soft tissue tumors, 2nd edn. Springer-Verlag, Padova, pp 179-196

2. Porter DE, Simpson AH (1999) The neoplastic pathogenesis of solitary and multiple osteochondromas. J Pathol 188:119-125

3. Stieber JR, Pierz KA, Dormans JP (2001) Hereditary multiple exostoses: a current understanding of clinical and genetic advances. Univ PA Orthop J 14:39-48
4. Garrison RC, Unni KK, McLeod RA et al (1982) Chondrosarcoma arising in osteochondroma. Cancer 49(9):1890-1897

5. Ahmed AR, Tan TS, Unni KK et al (2003) Secondary chondrosarcoma in osteochondroma: report of 107 patients. Clin Orthop Relat Res 411:193-206

6. Solomon L (1974) Chondrosarcoma in hereditary multiple exostosis. S Afr Med J 48(16):671-676

7. Pierz KA, Womer RB, Dormans JP (2001) Pediatric bone tumors: osteosarcoma, Ewing's sarcoma, and chondrosarcoma associated with multiple hereditary osteochondromatosis. J Pediatr Orthop $21: 412-418$

8. Mirra JM (1989) Benign cartilaginous exostoses: osteochondroma and osteochondromatosis. In: Mirra JM (ed) Bone tumors: clinical, radiologic and pathologic correlations Vol 2. Lea \& Febiger, Philadelphia, pp 1626-1659

9. Lange RH, Lange TA, Rao BK (1984) Correlative radiographic, scintigraphic, and histological evaluation of exostoses. J Bone Joint Surg Am 66(9):1454-1459

10. Kenney PJ, Gilula LA, Murphy WA (1981) The use of computed tomography to distinguish osteochondroma and chondrosarcoma. Radiology 139(1):129-137

11. Lee JK, Yao L, Wirth CR (1987) MR imaging of solitary osteochondromas: report of eight cases. AJR Am J Roentgenol 149(3):557-560

12. Aoki J, Sone S, Fujioka F et al (1991) MR of enchondroma and chondrosarcoma: rings and arcs of Gd-DTPA enhancement. J Comput Assist Tomogr 15(6):1011-1016

13. De Beuckeleer LH, De Schepper AM, Ramon F (1996) Magnetic resonance imaging of cartilaginous tumors: is it useful or necessary? Skeletal Radiol 25(2):137-141

14. Bottner F, Rodl R, Kordish I et al (2003) Surgical treatment of symptomatic osteochondroma. A three- to eight-year follow-up study. J Bone Joint Surg Br 85(8):1161-1165

15. Arkader A, Dormans JP, Gaugler R et al (2007) Spontaneous regression of solitary osteochondroma: reconsidering our approach. Clin Orthop Relat Res 460:253-257

16. Wiater JM, Farley FA (1999) Popliteal pseudoaneurysm caused by an adjacent osteochondroma: a case report and review of the literature. Am J Orthop 28:412-416

17. Vasseur MA, Fabre O (2000) Vascular complications of osteochondromas. J Vasc Surg 31(3):532-538

18. Tobias AM, Chang B (2004) A rare brachial artery pseudoaneurysm 13 years after excision of a humeral osteochondroma. Ann Plast Surg 52(4):419-422

19. Gerrand CH (1997) False aneurysm and brachial plexus palsy complicating a proximal humeral exostosis. J Hand Surg [Br] 22(3):413-415

20. Villanueva-Garcia E, Bas-Hermida P, Espinosa-Lledo C (1995) Pseudoaneurysm of the brachial artery caused by an osteochondroma: a report of two cases. Int Orthop 19:248-250

21. Koenig SJ, Toth AP, Martinez S et al (2004) Traumatic pseudoaneurysm of the brachial artery caused by an osteochondroma, mimicking biceps rupture in a weightlifter: a case report. Am J Sports Med 32(4):1049-1053

22. Brisse H, Orbach D, Klijanienko J et al (2006) Imaging and diagnostic strategy of soft tissue tumors in children. Eur Radiol 16(5):1147-1164

23. Bacci G, Balladelli A, Forni C et al (2007) Ewing's sarcoma family tumours. Differences in clinicopathological characteristics at presentation between localised and metastatic tumours. J Bone Joint Surg Br 89(9):1229-1233 